\title{
A Comparative Study on Emerging Modern and Traditional Rich people Lifestyles in Tehran
} City (Iran)

\author{
Nassim Nematizadeh \\ PhD student in Islamic Azad university Tehran \\ nasimnnz@yahoo.com \\ Solmaz Chamani \\ PhD student in Islamic Azad university Tehran \\ s.chamani1985@yahoo.com \\ Shabab Abbasi Geravand \\ Researcher \\ shababkhan2002@yahoo.com
}

DOI:10.5901/mjss.2014.v5n22p450

\begin{abstract}
This study was compiled in measuring method and in the statistical population of all the family guardians of Tehran city residences in districts 1,2, and 3 in year 2010, and in a sample of 397 people, who were chosen in multi-step cluster sampling method, to achieve the purposes of three main and eleven sub theories. The required information was collected using a 59itemed questionnaire with a proper reliability and validity between 0.72 to 0.79 for the whole questionnaire and its components, while the data were statistically analyzed using the descriptive statistics (frequency tables, central bias, distribution indexes and chart), and inferential statistics methods (Man Whitney). The study findings show that: (1) there is a meaningful difference between the main theories of emerging and traditional rich people lifestyles $(p<0.01)$; (2) there is a meaningful difference between the type of economic structures of emerging and traditional rich people lifestyles $(p<0.01)$; (3) there is a meaningful difference between the type of emerging and traditional rich people lifestyles $(p<0.01)$. Also regarding studies on eleven sub theories it was revealed that: (4) there is a meaningful difference among the level of education, belief in chance, belief in body management, way of passing leisure time, consumption model, and work styles variables between the emerging and traditional rich people $(p<0.01)$; and (5) there is observed no meaningful difference among the taste, religious principles, belief in the position of women, financial criterion, type of clothing variables between the emerging and traditional rich people $(p>0.05)$.
\end{abstract}

Keywords: Lifestyle, Socio-cultural Structure, Economical Structure, Emerging Modern Rich people , Traditional Rich people

\section{Introduction}

Lifestyle is one of the discussions which highly focused by the scholars and practitioners during the past couple of decades. It is one of the social phenomena which enjoy a certain position in today's life. Some issues such as fashion, taste, lifestyle are methods which are applied both to make distinction between one and others, and provide one's need for social coping. Apart from their functions in the social classification system, these factors result in social dynamism, by connecting to which one may also promote within the social dynamism (Simmel, 1981, Bourdieu ,1984, Gronow, 1991). Among the respective practitioners, Anthony Giddens more emphasizes on the lifestyle identity giving of the lifestyle (Fazeli, 2003: 110). Gioddens (1999) believes that due to increased communications and personal interests in the modern societies, people choose their certain lifestyles among a variable set of lifestyles, and also the lifestyles visualize for others the certain narration which one has chosen for his/her personal identity for others (Giddens, 1999: 28). According to the Giddens idea with respect to the lifestyle issue, the lifestyle study includes the effort to realize a regulated set of behaviors with action models chosen by people, whose actions in their daily lives are directed by those models. It is important that such choices are available in any level, and no culture would avoid people from their choices completely (Fazeli, 2003: 66).

Style means as the type of doing something (Aryanpour, 2001: vol 1, 103). The word lifestyle indicates various methods of living. Also, this expression may indicate the comparison of the lifestyles discovered among different groups of the society. Also, the word lifestyle as the meaning of "way of living" has been conceptualized. Usually, way of living is realized via 
values and methods of consumption, which are resulted from the increasing distinction of the developed capitalist societies (Babaei, 2005: 15). The methods of life are in fact the distinct model of communicating among the people, while people use the concept of lifestyle in their daily lives without considering explanation of their purpose as necessary (Chini, 1996: 4-5). According to personality value viewpoint, Wales and Lycert (1979) believe that the lifestyle thinking is understood as the interfering variables between difficult economical conditions and real consumption behaviors, while considering the lifestyle characteristics as including the activities, interests and beliefs. Also Sobel (1981) believes that the lifestyle and societies social structures are related. However, the options for anybody are highly dependent on one's position inside such structure (Gibbons and Bourimer, 2002: 113). These positions are formed based on the social opportunities structure and public availability of the opportunities, attributive characteristics such as gender, race, age and living change for the people and groups. According to Webber, lifestyle is applied in three meanings: style of life, lifestyle of action, life conduct. Lifestyle of action refers to the options of the people, while living chance is the potential to achieve the options (Dazeli, 2003: 28).

The lifestyle indicates one's actions within the scope of this life. in the traditional societies, the options based on consumption are highly dictated based on class, category, rural or family environments, while in the modern societies after all the people have more freedom in choosing the goods, services and actions which create the social identity (Solomon 1994: 438, quoted by Rasouli 2004: 54). Lifestyle is usually connected to social and economical class and is reflected in one's made image (Asaberger, 2000: 141).

According to Webber, apart from establishing the differences among the groups, the lifestyles explain the superiority of positions and classes. These groups are converted into reference groups via lifestyles and also define the social judgment and ethical assessment criteria. This thought has seriously been followed-up in Peir Bourdieu works (1980). In this regard, Bourdieu has referred seven concepts of field, capital, habituate, practice, symbol, class, taste in lifestyle analysis (Fazeli 2003: 37).

Apart from Robert discussions (1999) and his other supporters, it seems that lifestyle has been regenerated in these years (Rimmer ,1995). The changes made since Marx until today's era in capitalism and passing through the production to consumption step and besides that relative improvement of middle and lower classes in Europe and USA and growth and development of middle class have made a kind of personal independence in practice and personal freedom to choose (Chani ,1996:4). During the recent years, lifestyle is not considered in studies of social classification and as a way to determine the social class, but a modern social formation which is merely meaningful within the cultural changes of the modern era and consumption culture growth and development (Chavoshian ,2004: 6). Today, the concept of lifestyle has highly been changed due to changes of the western capitalist system in the works of the social sciences thinkers, so that from the prestige models which are totally dependent on the economical components, materialistic valu4s and wealth, and study the lifestyles in certain classes to the models based on the consumptions of cultural and art goods and way of passing leisure times and have different shapes in various social classes and groups are included herein (Chenni ,1999: 19).

Various factors affect lifestyle (Moradi, 2004: 229). The lifestyles are considered as a set of actions and orientations which are meant in certain basis and are dependent on cultural states. Lifestyle is a state and way to use the goods, locations, and certain times which identify a certain group. However, it may not express the whole social experience of the same (Chini ,1996: 4,5). In the classi and contemporary sociologists works, the lifestyles with two dependent and independent variables may also be indicated. In the sociology literature in the primary formulation which dates back to 1920's, lifestyle, wealth consumption and social position of people have mainly been used as an index to determine social class (Abazari and Chavoshian, 2002: 6, quoted from Chaein, 1955, Chapman 1935). In second formulation, the lifestyle in the social classification studies is not considered as a way to determine the social class, but is considered as a modern social form which is merely meant within the cultural changes of the modern era and consumption culture growth and development (Chavoshian, 2004:6). In this regard, the lifestyle is a way to define the values, visions, behaviors or identities of people which is of increasing importance for social analysis (Abazari \& Chavoshian, 2002: 6).

Whereas theoretically an important part of this study is referred to the emerging rich people, in order to understand the concept of emerging rich peoplees in Iran, the reasons to apply the word is mandatory. In definition of class, Bokhinsky (1995) wirtes: classes are groups of people from whom one may possess the other's job following in his position change in a certain socio-economical system. Kohen et al (1996) state in defining the social class that it is a group who are defined based on their relation with the ownership and control on the production factors and on others' manpower (Rafipour, 1999). These definitions show that classes are in two formats, i.e. are in two distinct groups. Thus, their classification is made nominally. Lipist et al classify all the social class definitions in two groups 1. realistic definitions 2. nominal definitions of the social class. In realistic definitions, they address three major criteria: (1) mutual action (social class as a group who have mutual actions distinctive to other classes members) (2) members class knowledge from the class categorization from the 
economical and political interests and membership in a class (3) different class culture with a certain lifestyle for each of the social classes, Shumpiter (1919-1927), Makyor and Peach (1949) and Pasovsky (1957) emphasize in these bases (Lipist et al, 2992: pp 130, 131). Also Lensky (1966) has addressed in his studies that due to the limited valuable sources in each historical era, we would like to know why some rich people have more shares in the style of such sources. The response to these questions and many other questions means talking about the existence and socio-economical, political and cultural results of social class, social grouping, social inequality and their effects on the public actions of life.

Since the beginning, the thinkers have shown certain interest on social grouping and have pointed out the inherent and sustainable features of such conditions while addressing their role in the social life. Aristotle talked about and Plato studies these conditions (Darenbdrov ,1968: 153). Plato addressed the classified society discussion in making his new society based on justice, and internal social stability (Taamin, 1994:25).

Among different groups of people, this study focused on rich people. Here, rich people mean those who have power purchase higher than the costs of the essential goods and issues and comforting needs on their demand, while their preferences are also achieved. Thus, the indexes to be rich are 1. power of buy demands and 2. achieved preferences. The purchase power is analyzed in micro level cultural aspect, i.e. lifestyle. In Iran, rich people include the top and rich middle classes. In this study, the emerging rich people are those who have recently ascended from lower to higher social layers via inter- or intra- generation class dynamism and became rich. That is, they have the purchase power for their various needs and demands.

Tony Fitz Petrick in the book titled the theory of comfort, analyzed and criticizes some five indexes to define comfort in micro level. These five indexes, each of which are focused on certain aspects of comfort, include: (1) happiness, i.e. the satisfaction and happiness due to achievement of wishes. While sometime being rich may result in happiness (2) security, i.e. one benefits from security, income, employment and housing. Security means prior knowledge on that one's life will not be subject to disorder in near future. (3) preference, a person whose preferences are achieved has more comfort than the others. In case I wish to buy a new car and may pay for it, then my satisfaction and comfort level are higher than one who wishes to buy such car but does not have the money. The importance of this definition is its measurability. (4) needs, all the types of needs achievements may not be thought to be equal to comfort according to importance. There shall be considered difference between the needs. a. fundamental needs, b. non-fundamental needs, c. preferences. fundamental needs may be considered to be connected to the comfort. However, the other two classes are not essentially of such position. (5) rightness. Rightness refers to the a balanced point between partnership and reward. For instance, in case I participate double as you in an investment project, then my dividend shall be double as yours. In case I get more than that, it will be injustice against you, and if less, then I will be subject to injustice. (6) relation comparison, in real world, the comfort level of each person is totally dependent on the level of comfort of those of his type, i.e. those to whom he is comparing himself. Comfort is in a way linked to this feature of one who always compares himself with others (Tony Fitz Petrik, 2002: 20-30).

Regarding the positions and features of emerging, Max Webber says: according to the class position, facilities and economical life opportunities, the recent rich are in the same position of the top groups. However, these groups are not totally acceptable of highly prestigious groups. In fact, these groups are acting as trainees to be introduced to the features of high prestigious group. In order to withdraw emerging people, the highly prestigious people uses various excuses. For instance, they state that emerging have not enough education, of low information and knowledge, or that they do not know how to spend their money, or at last but not least they state that their parents are not known (Khodabandelou, 19994: 33, quoted from Henry Menderas 1990).

Lloyd Warner believes that the top down group of people are wealthy, and even often wealthier than the top of the tops class; but they have become rich recently and may even be too recently. They do not have proper family background, these two factors have separated them from top class of the society. According to the income, residence and level of social participation, top-down class are like top of the tops class, but they do not have the proper glory and correct tastes and acceptable social procedure (Adibi 1975, 226, 227).

According to the aforesaid, lifestyle is one of the discussions which have been considered in various fields especially sociology during the recent decades, and some studies have been made regarding the modern and traditional rich people lifestyles, however not enough. Thus, it has been tried to fulfill researching on this issue and clarify the same sociologically. The main question here is that whether there is any difference between the lifestyles of modern emerging and traditional rich classes in Iran, and how it is in their lifestyle, and what is these two classes behavioral model? Is there any behavioral difference between these two groups? How is the clarification this difference sociologically? How is its appearance in their consumption model? What are its aspects and structure? How are its experimental effects? 


\section{Method}

This study was compiled in measuring method and in the statistical population of all the family guardians of Tehran city residences in districts 1,2, and 3 in year 2010. According to the statistics obtained from Tehran (Shemiranat) Municipality General Administration, these three districts have 21 counties, with a population of 1,646,076 in 485,873 families; a sample of 397 people were chosen as statistical sample in multi-step cluster sampling method, to achieve the purposes of three main and eleven sub theories. The sampling method in practice was so that first of all some of the aforementioned districts counties were chosen randomly and then some of the family guardians were chosen as the statistical sample.

In this study, a questionnaire made by the author was used which contained 59 questions with proper reliability and validity as the information collection tool. In validity study, the same was obtained between 0.72 and 0.79 using the Cronbakh Alpha factor, for questionnaire and its components. Also in information collection, by referring to the houses during 20 days, approximately 20 questionnaires were completed per day (except holidays) by four surveyors, who were scholar master's degree students who enjoyed the required explanations and training to complete such questionnaires and there was no need to give information to them. Also in statistical analysis, the central bias indexes and distribution and indexes and charts have been used in descriptive level; while in the inferential level the Man Whitney test have been used to study the research theories.

\section{Study Findings}

The findings of study and testing the theories indicate the following:

(1) There is a meaningful difference between the main theories of emerging and traditional rich people lifestyles $(p<0.01$; so that it may be said there is differences between these two groups according to the education, believe in chance, body management and way to pass leisure times (Table 1).

(2) There is a meaningful difference between the type of economical structures of emerging and traditional rich people lifestyles $(p<0.01)$; so that it may be said there is differences between these two groups according to their type of consumption model (Table 1).

(3) There is a meaningful difference between the type of emerging and traditional rich people lifestyles $(p<0.01)$; so that it may be said there is differences between these two groups according to their lifestyles (Table 1).

Table 1- Man Whitney Test Results in Main Theories Study

\begin{tabular}{|l|l|l|l|l|}
\hline Theory & Compared variable & Rich people & Average rank & Meaningful level \\
\hline \multirow{2}{*}{1} & \multirow{2}{*}{ Social and cultural structure } & Traditional & 210.45 & \multirow{2}{*}{.000} \\
\cline { 3 - 4 } & & Modern & 143.63 & \\
\hline \multirow{2}{*}{2} & \multirow{2}{*}{ Economical structure } & Traditional & 204.31 & \multirow{2}{*}{.000} \\
\cline { 3 - 4 } & & Modern & 173.29 & \multirow{2}{*}{.005} \\
\hline \multirow{2}{*}{3} & \multirow{2}{*}{ Lifestyle } & Traditional & 202.24 & \\
\cline { 3 - 4 } & & Modern & 183.31 & \\
\hline
\end{tabular}

(1) in studying the first sub theory, as "there is a meaningful difference between the emerging and traditional rich people regarding level of education", the results approve the same, so that the level of education of modern group was higher than the same for the traditional group $(p<0.01)$.

(2) in studying the second sub theory, as "there is a meaningful difference between the emerging and traditional rich people regarding taste", the results reject the same, $(p>0.05)$.

(3) in studying the third sub theory, as "there is a meaningful difference between the emerging and traditional rich people regarding religious principles", the results reject the same, $(p>0.05)$. 
(4) in studying the fourth sub theory, as "there is a meaningful difference between the emerging and traditional rich people regarding believe in chance", the results approve the same, so that the orientation to superstition of modern group was higher than the same for the traditional group $(p<0.01)$. (table 2$)$

(5) in studying the fifth sub theory, as "there is a meaningful difference between the emerging and traditional rich people regarding belief on women position", the results reject the same, $(p>0.05)$. (table 2$)$

(6) in studying the sixth sub theory, as "there is a meaningful difference between the emerging and traditional rich people regarding believe in body management", the results approve the same, so that the orientation to body management of modern group was higher than the same for the traditional group $(p<0.01)$. (table 2 )

(7) in studying the seventh sub theory, as "there is a meaningful difference between the emerging and traditional rich people regarding the way to pass leisure time", the results approve the same, $(p<0.01)$. (table 2 )

(8) in studying the eighth sub theory, as "there is a meaningful difference between the emerging and traditional rich people regarding the consumption model", the results approve the same, $(p<0.01)$. (table 2$)$

(9) in studying the ninth sub theory, as "there is a meaningful difference between the emerging and traditional rich people regarding financial criterion", the results reject the same, $(p>0.05)$. (table 1$)$

(10) in studying the tenth sub theory, as "there is a meaningful difference between the emerging and traditional rich people regarding way of clothing", the results reject the same, $(p>0.05)$. (table 2$)$

(11) in studying the eleventh sub theory, as "there is a meaningful difference between the emerging and traditional rich people regarding the type of working style", the results approve the same, $(p<0.01)$. (table 2$)$

Table 2- Man Whitney Test Results in SubTheories Study

\begin{tabular}{|c|c|c|c|c|}
\hline Theory & Compared variable & Rich people & Average rank & Meaningful level \\
\hline \multirow[b]{2}{*}{1} & \multirow{2}{*}{ Three- level academic degree } & Traditional & 167.16 & \multirow{2}{*}{.004} \\
\hline & & Modern & 135.83 & \\
\hline \multirow{2}{*}{2} & \multirow{2}{*}{ Taste } & Traditional & 195.24 & \multirow{2}{*}{.097} \\
\hline & & Modern & 217.18 & \\
\hline \multirow{2}{*}{3} & \multirow{2}{*}{ Religious principles } & Traditional & 198.17 & \multirow{2}{*}{.194} \\
\hline & & Modern & 203.00 & \\
\hline \multirow{2}{*}{4} & \multirow{2}{*}{ Believe in superstition } & Traditional & 200.06 & \multirow{2}{*}{.000} \\
\hline & & Modern & 170.66 & \\
\hline \multirow{2}{*}{5} & \multirow{2}{*}{ Believe in women position } & Traditional & 183.62 & \multirow{2}{*}{.106} \\
\hline & & Modern & 161.62 & \\
\hline \multirow{2}{*}{6} & \multirow{2}{*}{ Believe in body management } & Traditional & 203.69 & \multirow{2}{*}{.000} \\
\hline & & Modern & 103.50 & \\
\hline \multirow{2}{*}{7} & \multirow{2}{*}{ Passing leisure times } & Traditional & 191.97 & \multirow{2}{*}{.002} \\
\hline & & Modern & 233.03 & \\
\hline \multirow{2}{*}{8} & \multirow{2}{*}{ Consumption model } & Traditional & 209.52 & \multirow{2}{*}{.000} \\
\hline & & Modern & 148.09 & \\
\hline \multirow{2}{*}{9} & \multirow{2}{*}{ Financial criterion } & Traditional & 179.40 & \multirow{2}{*}{.950} \\
\hline & & Modern & 180.04 & \\
\hline \multirow{2}{*}{10} & \multirow{2}{*}{ Type of clothing } & Traditional & 199.75 & \multirow{2}{*}{.722} \\
\hline & & Modern & 195.36 & \\
\hline
\end{tabular}




\begin{tabular}{|l|l|l|l|l}
\hline \multirow{2}{*}{11} & \multirow{2}{*}{ Work style } & Traditional & 206.06 & \multirow{2}{*}{.000} \\
\cline { 3 - 4 } & & Modern & 131.48 &
\end{tabular}

\section{Discussion and Conclusion}

The most important findings of this study focused in comparison of the two traditional and modern rich people, indicate the existence of meaningful differences between the socio-cultural, economical structures and lifestyles of these two groups.

In order to clarify these findings as per the reliable sociology theories, it may be said that in fact lifestyles include models for the behaviors which distinct people from each other. Mainly, different life models are positioned in culture expression and also culture may be explained so that it is a set of people lifestyles including their common customs, visions, values and understanding, which link then as a society to another. Socio-cultural structure includes a set of variables such as high education, taste, etc.

According to Weblen, high education was primarily of the tasks of the traditional lazy classes, however, after industrial societies' progress, the orientation with respect to high education was changed. Due to the findings of this study, the modern rich people were more educated than the traditional rich people.

According to Bourdieu, the difference between today and past generation as per the taste criteria is because the economical status has changed; thus, due to the existence of public communication means, there basis to grow taste was developed which is also including various criteria. Due to the findings of this study, even the traditional rich people's tastes are being modernized.

According to Weblen, observation of religious orders is a way of thinking which has certain economical value, and is assessed via the effect of the same on one's profitability and his industrial and production efficiency, which may be different for various classes; however, Due to the findings of this study, the religious beliefs in these two groups are almost the same.

Also Weblen suggests that brilliance and type of clothing indicate laziness, especially for the recently industrialized societies emerging layers, the type of clothing and wearing are important. Due to the findings of this study, we understand that the way of shopping and type of clothing is moving towards modernity among rich people.

"In fact, these groups are acting as trainees to be introduced to the features of high prestigious group. In order to withdraw emerging people, the highly prestigious people uses various excuses. For instance, they state that emerging have not enough education, of low information and knowledge, or that they do not know how to spend their money; or at last but not least they state that their parents are not known "says Max Webebr regarding the position and features of the emerging rich people. Classification of people in prestigious classes is mainly based on their consumption model. Prestigious groups are mainly those people related to the lifestyles and social respect. These people consider limits for their relations with people not belonging to their groups and expect its members to have social distance from those of lower prestige; however, according to this study, the tradition group members are also moving towards modern consumption model.

Due to the findings of this study, it may be concluded that the mental contradiction and gap is one of the Iranian social gaps and contradictions occurred between tradition and modernism. It seems that tradition is under the process of regeneration and that the traditionalists shall avoid some their positions and these people are more modern than their ancestors.

Evidently, lack of cultural integrity is the result of the process and regeneration of a society, which has affected the old values and interests and brings new interests and values.

In the third world societies, the passing era from the traditional to modern society is considered by the sociologists and social sciences practitioners due to their effects on the social structure. In this regard, like many other developing countries, our country is affected by the passing era issues in the fields of culture, values and social structure.

\section{References}

Aryanpour ( 2001), Dictionary: vol 1, 103

Asaberger,A.(2000). Media Analysis Techniques, translated by parviz ajlali, Centre for Research and Media Studies tehran. Abazari ,u .\& Chavoshian,H.( 2002), Of social class and lifestyle. Journal of Personality and Social sience ,n20 . 
Adibi,H ( 1975), New middle class in Iran,pablished by sociology

Babaei ,M( 2005), Investigate the relationship between socioeconomic status and lifestyle of the upper classes in Tehran, Sociology Theses in university azad olom tahghighat in iran ,tehran.

Boukhinsky 1995

Bourdieu( 1984), outline of theory of practice "Cambridge university press" .

Bourdieu,(1980), Theory of Action. Translated by morteza mardiha, published by naghshe negar .

Pasovsky 1957

Taamin,M.( 1994), Sociology, social stratification and inequality, and the theoretical. Translated by abdolhosein nikgohar ,tehran :published by totia

tony Fitz Petrik 2002

Chavoshian,H.( 2004), Lifestyle and social identity.tehes phd university tehran.

Chini ,D.(1999) ,lifestyle , translated by chavoshi ,A.tehran , Ministry of Culture and Islamic Guidance

Chini ,D (1996). The Same, 23.

Khodabandehlou,S.( 1994), The sociology of class and social inequality, published by jahade daneshgahi.

Darendrof,R.( 1968) , « Social beings, translated by gholamreza khadivi ,published by agah

Robert 1999

Rasouli 2004

Rafipour,F.( 1999), Investigation and conception, published by sahami nashr.

Rimmer 1995

Samuel 1981

Sobel 1981

Shompiter, . (1919-1927)

Fazeli,M.( 2003), consum and lifestyle . published by : nashre sobhdam.

Kohen 1996

Gronow,G.( 1991), sociology of teste, routeledge.

Gibbons and Bourimer 2002

Giddens ,A. (1999) , Consequences of modernity , translated by mohsen salasi , published by: nashremarkaz

Giddens ,A. (2005), World Vision , translated by mohamadreza jalalipor, published by : tarheno

Lensky,G.(1977), sociology and sociology : an alternative view " the american sociologist ,12: 72 _75.

Lipist et al( 20020

Makiorovic (1949)

Moradi ,A. (2004) , Media's role in creating a culture for export development, these phd university azad olom tahghighat in tehran.

Moein , M. ( 1992) , Persian culture.published by: amirkabir

Wales and Lykert, ( 1979 ), sociology for economy, translated by :hadi samadi , published by :samt 